
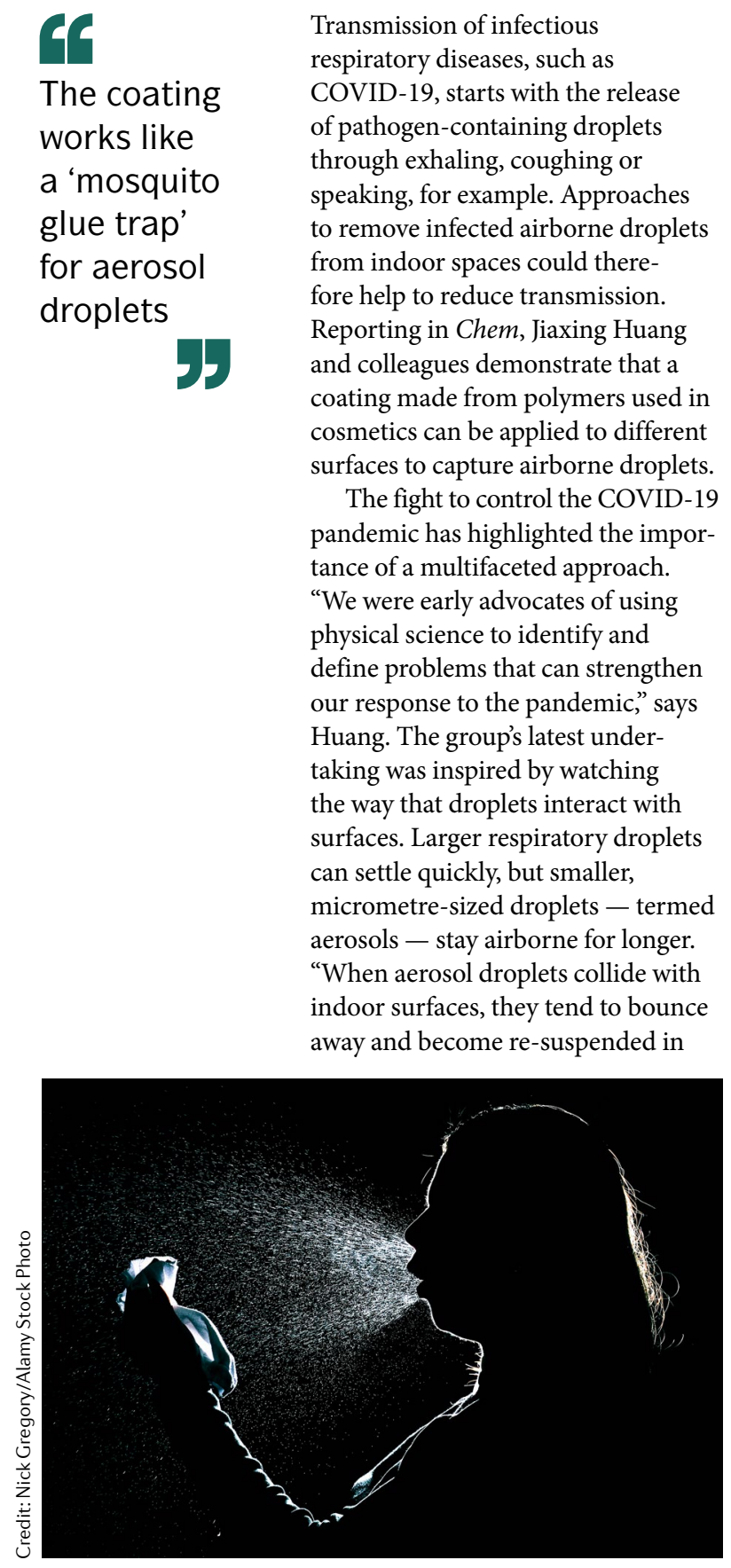

Transmission of infectious respiratory diseases, such as COVID-19, starts with the release of pathogen-containing droplets through exhaling, coughing or speaking, for example. Approaches to remove infected airborne droplets from indoor spaces could therefore help to reduce transmission. Reporting in Chem, Jiaxing Huang and colleagues demonstrate that a coating made from polymers used in cosmetics can be applied to different surfaces to capture airborne droplets.

The fight to control the COVID-19 pandemic has highlighted the impor"We were early advocates of using physical science to identify and define problems that can strengthen our response to the pandemic," says Huang. The group's latest undertaking was inspired by watching the way that droplets interact with surfaces. Larger respiratory droplets can settle quickly, but smaller, micrometre-sized droplets - termed aerosols - stay airborne for longer. "When aerosol droplets collide with indoor surfaces, they tend to bounce away and become re-suspended in

\title{
Surface coating captures droplets
} tance of a multifaceted approach. capacity needed in many public
settings. Moreover, the coated slide remained transparent and haze-free - a beneficial property for protective barriers, such as acrylic screens and face shields.

The next step was to evaluate the performance of the coating on exposure to larger and faster droplets that more closely simulate those emitted during violent respiratory events, such as coughing or sneezing. Using an air-spray gun filled with $1 \%$ aqueous $\mathrm{NaCl}$ and a red dye, the team directed a droplet stream at coated and uncoated acrylic screens. White paper placed at the bottom of each screen to detect escaped droplets revealed that the coating reduces the number of rebounded droplets by $80 \%$. "The coating works like a 'mosquito glue trap' for aerosol droplets," says Huang. "The polyelectrolyte absorbs water droplets and spreads them out, dissipating their kinetic energy so that they can no longer contract and jump."

The coating formulation was optimized by adding alkyl polyglycosides - non-ionic, plantderived surfactants commonly used in personal-care products. The alkyl polyglycosides increase the wettability of the solution, so that the coating can be applied to materials with different surface chemistries and textures - ranging from cloth to concrete - and modify the viscosity, making the solution more tolerant of additives, including dyes and sanitization agents, such as $\mathrm{Cu}^{2+}$.

Although their concept has not yet been tested using real human respiratory droplets, Huang points out that the droplet concentrations tested were much higher, so their coating should work even better in a real scenario. The team are now eager to test this hypothesis.

Claire Ashworth

ORIGINAL ARTICLE Yu, Z. et al. Droplet-capturing coatings on environmental surfaces based on cosmetic ingredients. Chem https://doi.org/ 10.1016/j.chempr.2021.05.017 (2021) RELATED ARTICLES Huang, H. et al. COVID-19: a call for physical scientists and engineers. ACS Nano 14, 3747-3754 (2020) | Jabbour, C. R. et al. Chemical targets to deactivate biological and chemical toxins using surfaces and fabrics. Nat. Rev. Chem. 5, 370-387 (2021) 